\title{
CONF-94/2/6--2
}

\section{LOW LEVEL MONITORING AND CONTROL OF NONLINEAR SYSTEMS}

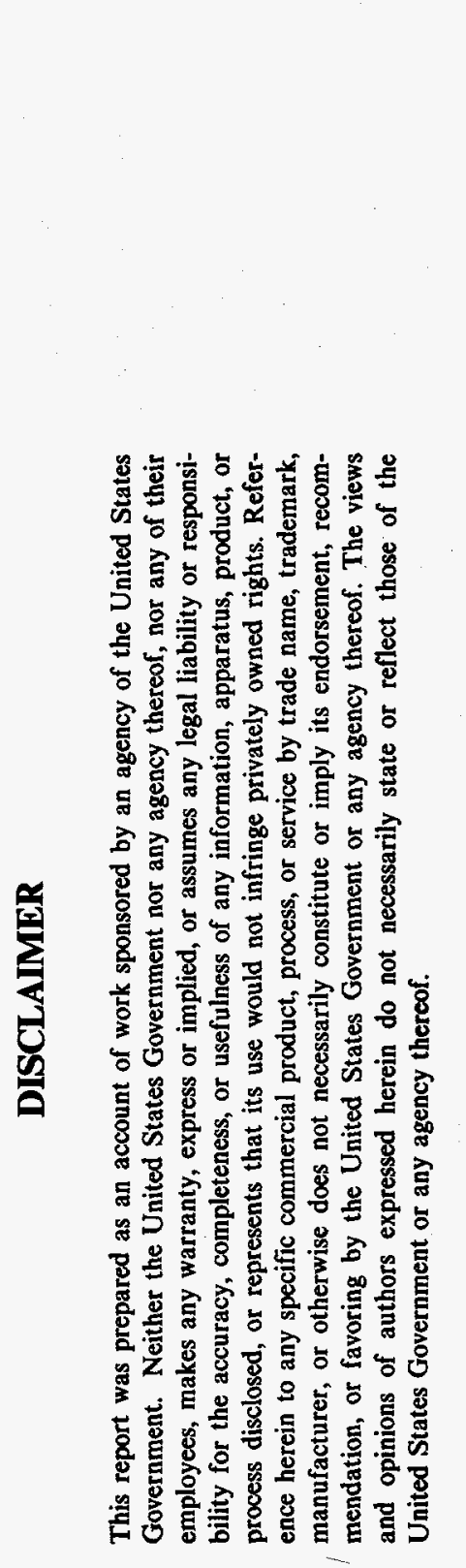

\author{
Alan Cover \\ Clemson University, Dept of Math. Sciences \\ Clemson, S. C. $29634-1907$ \\ Suzanne Lenhart \\ University of Tennessee, Math. Department \\ Knoxville, TN 37996 \\ Vladimir Protopopescu* \\ Oak Ridge National Laboratory \\ Engineering Physics and Math. Division \\ Oak Ridge, TN 37831-6363 \\ James Reneke \\ Clemson University, Dept of Math. Sciences \\ Clemson, S.C 29634-1907
}

To be presented at the 33rd IEEE Conference on

Decision and Control

Lake Buena Vista, FL

December 14-16, 1994

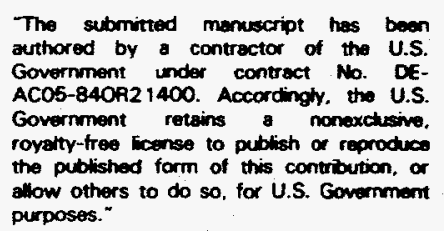

"Research sponsored by the US Department of Energy at ORNL under contract DE-AC05-84OR21400 with Martin Marietta Energy Systems, Inc. 


\section{DISCLAIMER}

Portions of this document may be illegible in electronic image products. Images are produced from the best available original document. 


\title{
33RD CDC \\ LOW LEVEL MONITORING AND CONTROL OF NONLINEAR SYSTEMS
}

ALAN COVER

CLEMSON UNIVERSITY, DEPT. OF MATH. SCIENCES, CLEMSON, S.C. 29634-1907

SUZANNE LENHART

UNIVERSITY OF TENNESSEE, MATHEMATICS DEPARTMENT, KNOXVILLE, TN 37996

VLADIMIR PROTOPOPESCU

OAK RIDGE NATIONAL LABORATORY, ENGINEERING PHYSICS \& MATH DIVISION, OAK RIDGE, TN 37831-6363

\author{
JAMES RENEKE \\ CLEMSON UNIVERSITY, DEPT. OF MATH. SCIENCES, CLEMSON, S.C. 29634-1907 \\ E-MAIL: RENEKE @ CLEMSON.BITNET, FAX: 1-803-656-5230
}

Key words. Monitoring, control, nonlinear systems

Monitoring and controlling separate components of large complex systems without overburdening or even involving a central supervisor is an outstanding task of smart engineering. Typical situations are: adjusting the position of an axle, steering a wheel, or closing and opening a valve. Even such innocuously simple tasks require robust and efficient stand alone sensors and actuators. The range of application of these smart sensors and actuators is greatly enhanced when the monitoring and controlling functions are based on generic, nonparametric methods.

In this paper, we propose a nonparametric method for monitoring and controlling nonlinear systems whose dynamics is, in general, unknown or only partially known. Our nonparametric method is based on the stochastic linearization of the underlying (unknown) nonlinear system.

The information about the nonlinear system is collected from component observations accumulated in sample covariance functions. A finite dimensional matrix representation of the linearized input/output operator is given by products of Choleski factors of discrete covariance of input and output processes.

The smart component monitoring function reduces to measuring the distance between the linearization of the actual system (assumed to be slowly evolving in time) with that of a "reference" system. The smart component control function is exercised by updating the linear feedback components via a nonparametric version of the linear quadratic regulator (LQR) [5].

The nonparametric method presented here will be compared with standard parametric methods [4] that proceed by fitting the known nonlinear model with a linear model, and solving the LQR problem. 


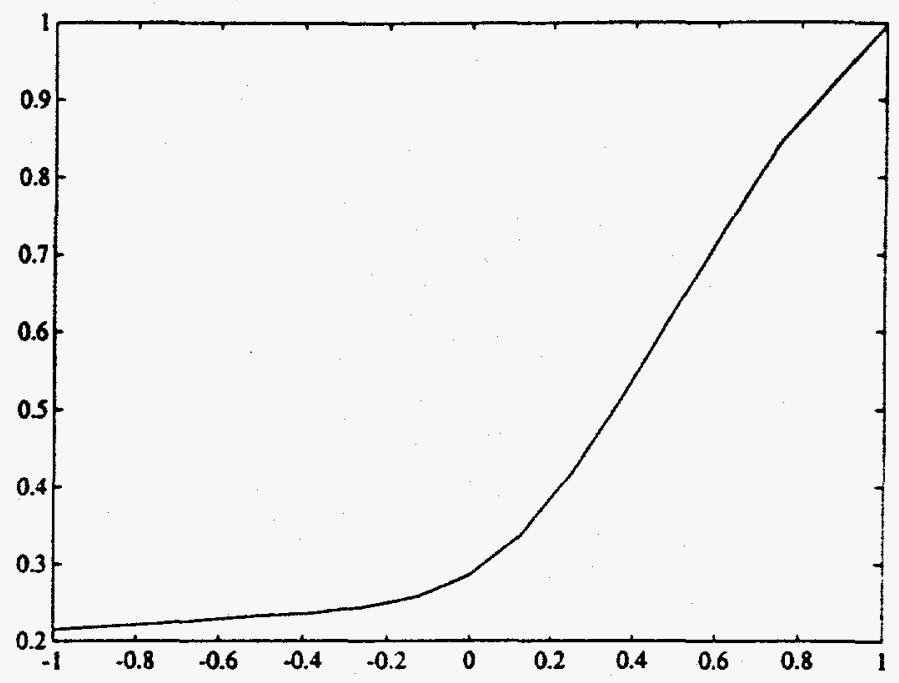

Fig. 1. Parameter a vs. The Distance from a Reference System

To describe our method, consider the nonlinear system $N$

$$
d x=N(x) d t=(A x+F(x)) d t
$$

where $A$ and $F$ are its linear and nonlinear parts, respectively. Since the system is, in principle, unknown, we perturb it by additive "white noise"

$$
d X=A X d t+F(X) d t+d W(t)
$$

where $W$ is the standard Wiener process [2]. We will use ' $X$ ' for stocahastic systems and ' $x$ ' for deterministic systems. The random perturbation would allow the system to explore larger regions of the phase space and provide more information about its dynamical behavior.

Given observations or data from monitoring,

$$
Y=C X
$$

over a sufficiently long period of time, we construct a covariance function $R(s, t)$ corresponding to the observation process, $Y(t)$

$$
R(s, t)=E(Y(s) Y(t)) .
$$

For background related to this construction and the one to one correspondence between processes and covariances, we refer to Parzen [3]. The mean of $Y$ and the covariance $R(s, t)$ will from now on, be supposed to contain all the available information about the nonlinear system.

Using techniques based on the theory of reproducing kernels in Hilbert spaces [6], an essentially unique linear system $L$

$$
\begin{gathered}
X=L W \\
2
\end{gathered}
$$


is derived, with $R(s, t)$ given by (4) as its covariance.

For this linear system we consider now the control problem

$$
X=L(W+u)
$$

with control $u$ and performance criterion

$$
J(u)=E(X(\infty))^{2}+c E(u(\infty))^{2} .
$$

The optimal control $u^{*}$ that minimizes the functional $J$ will then be applied as a feedback control to the original nonlinear system, in place of the stochastic disturbance $d W$

$$
d x=A x d t+F(x) d t+d u^{*}
$$

The efficiency of the control procedure is measured by closeness to a "reference" system.

To compute the covariances, it seems crucial that the nonlinear system have bounded trajectories. Since the Wiener process will make the trajectories wander over much of the phase space, simple boundedness does not seem to be sufficient. We conjecture that the more precise notion of "point dissipative system" (PDS) is needed for this construction.

Definition: A system is point dissipative if there is a bounded region which every trajectory of the system eventually enters and remains within.

A simple sufficient criterion that implies the point dissipative property for a reasonable class of 2 or 3 -dimensional systems is:

$u^{T} A u<0$ for all nontrivial solutions of the equation $f(u)=0$ [1].

We remark here that the point dissipative property may be destroyed using linear feedback controls.

As an example consider the following nonlinear system perturbed by noise

$$
\begin{aligned}
d X_{1} & =\left(a X_{1}+X_{1} X_{2}\right) d t+d W \\
d X_{2} & =-\left(X_{2}+X_{1}^{2}\right) d t \\
Y & =X_{1}
\end{aligned}
$$

The system is point dissipative for all values of $a$. For $a \leq 0$ the origin is a global asymptotic stable point. For $a>0$ the origin is unstable and the system develops two point attractors.

Using a metric for measuring the distance between the linearization of a given system and the linearization of the reference system $a=-1$ we obtain Figure 1 plotting $a$ vs. distance. The graph shows something is happening at $a=0$ and we can flag it using a metric measuring the distance between nonparametric linearizations. 


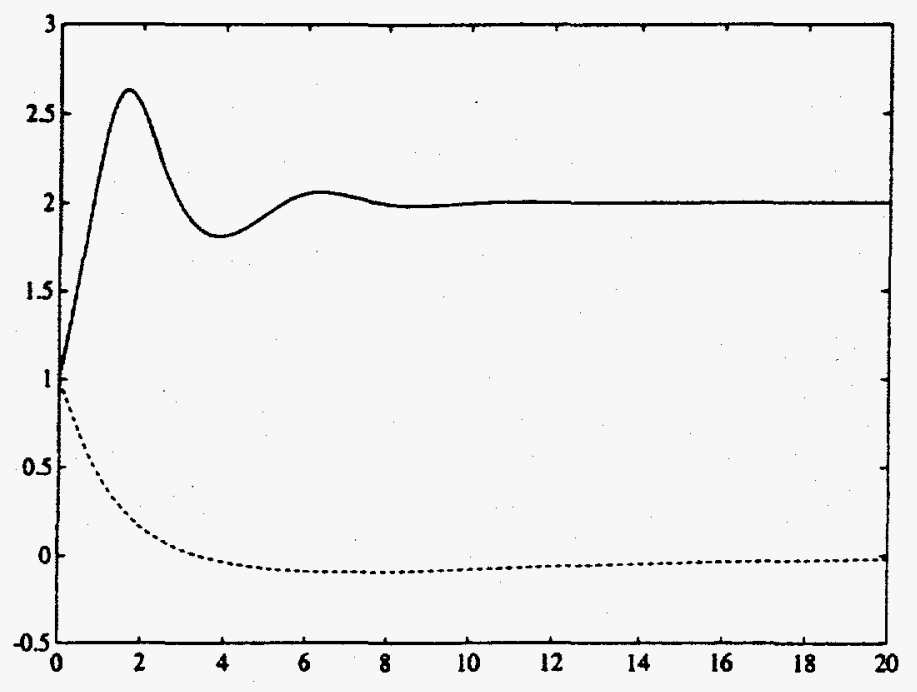

FIG. 2. Responses of the Uncontrolled and Controlled Systems

Figure 2 presents a comparison of a response for this system with $a=1$ and response for the system modified by a linear feedback obtained by solving the optimal control problem for a linearization of the system.

In conclusion we have shown that by using a nonparametric stochastic linearization of the underlying nonlinear system we can efficiently and economically carry out monitoring and control functions for a general class of nonlinear systems.

\section{REFERENCES}

[1] A. K. Bose, A. S. Cover AND J. A. RENEKE, On point dissipative n-dimensional systems of differential equations with quadratic nonlinearity, Internat. J. Math. and Math. Sci., 16(1993), pp. 139-148.

[2] A. Friedman, Stochastic Differential Equations and Applications, Vol. 1, Academic Press, New York, 1975.

[3] E. PARzEN, An approach to time series analysis, Ann.Math.Stat., 32 (1961), pp. 951-989.

[4] J. B. Roberts aNd P. D. Spanos, Random Vibration and Statistical Lineariztion, John Wiley \& Sons, Chichester, 1990.

[5] D. L. Russell, Mathematics of Finite-Dimensional Control Systems; Theory and Design, Marcel Dekker, Inc., New York and Basel, 1979.

[6] H. L. WeINERT, ed., Reproducing Kernel Hilbert Spaces; Applications in Statistical Signal Processing, Hutchinson Ross Publ. Co, Stroudsburg, PA, 1982. 